\title{
Transformative therapy in hypophosphatasia
}

\author{
Nick Bishop, ${ }^{1}$ Craig F Munns, ${ }^{2,3}$ Keiichi Ozono ${ }^{4}$
}

Hypophosphatasia (HPP) is a rare, potentially life-threatening disease characterised by hypomineralisation of bones and teeth. ${ }^{1}$ The fundamental defect is reduced functional activity of the enzyme tissue-nonspecific alkaline phosphatase (usually just termed alkaline phosphatase [ALP] in clinical practice). Homozygous or compound heterozygous mutations in the ALPL gene result in the most severe phenotypes of HPP. However, dominant negative effects can also be observed when a single heterozygous mutation affects the binding site(s) of the ALP homodimer. ALP catabolises inorganic pyrophosphate (PPi), an inhibitor of bone mineralisation, and pyridoxal 5'-phosphate (PLP; major form of vitamin $\mathrm{B}_{6}$ ), allowing pyridoxal to cross the blood-brain barrier and enter pathways leading to neurotransmitter synthesis.

There are six clinical forms of HPP, four of which have clear relevance in paediatric practice-perinatal, benign prenatal, infantile and juvenile HPP. In some instances the adult form has unrecognised paediatric onset, while odonto-HPP simply affects the teeth. ${ }^{1}$ Bone-targeted enzyme-replacement therapy (ERT) with asfotase alfa (administered subcutaneously 3-6 times per week) is now available, having recently been approved in several regions. For those presenting early in life, such treatment has been shown to reduce mortality during the first year of life from $\sim 97 \%$ in perinatally presenting cases and close to $60 \%$ in cases presenting later in infancy to $\sim 10 \%$ overall.

In contrast to many other disorders treated with ERT, there is no accumulation of toxic metabolites. Thus, with ERT, many of the findings of HPP may be reversible. Nonetheless, many patients with perinatal-onset and infantile-onset HPP are critically ill. Even with ERT, improvements in bone mineralisation may

${ }^{1}$ Department of Human Metabolism, University of Sheffield, Sheffield, UK; ${ }^{2}$ Department of Endocrinology, The Children's Hospital at Westmead, Westmead, New South Wales, Australia; ${ }^{3}$ Department of Paediatrics and Child Health, University of Sydney, Sydney, New South Wales, Australia; ${ }^{4}$ Department of Pediatrics, Osaka University, Graduate School of Medicine, Suita, Osaka, Japan

Correspondence to Dr Nick Bishop, Department of Human Metabolism, University of Sheffield, Sheffield S10 2TH, UK; n.j.bishop@sheffield.ac.uk take weeks to months. In this regard, some affected infants require considerable periods of critical care support due to respiratory compromise before demonstrating marked clinical improvement. Such compromise reflects the mechanical inadequacy of gracile, poorly mineralised ribs that may have fractured, and in some instances, tracheobronchomalacia. In one case study, a patient diagnosed in utero as having perinatal lethal HPP demonstrated severe hypomineralisation and required immediate intensive respiratory care upon birth. Radiographic improvements were observed in rib mineralisation after 20 days of ERT, and by day 207 the patient was fully weaned from mechanical ventilation. ${ }^{2}$ These observations are supported by results from a recently published study in which continuous ERT resulted in improvements in bone mineralisation and respiratory outcomes, such that of the $76 \%$ of the children who required ventilatory support at baseline and survived, $75 \%$ could be weaned from this support. ${ }^{3}$ Some infants suffer convulsions due to lack of pyridoxine getting across the blood-brain barrier. Survival in infants who suffered convulsions and were treated with ERT was 77\%, compared with a $100 \%$ mortality rate in a set of historical control infants who were not treated. ${ }^{3}$

Raised intracranial pressure can arise secondary to craniosynostosis, which is common in those presenting early in life. Frequent monitoring of head circumference and inspection of the optic discs should be undertaken. In affected infants, gross and fine motor functions are delayed but show steady catch-up over time; cognitive function is usually within the normal range within 12 months of starting treatment. A benign prenatal form of HPP has been classified that is typically diagnosed on ultrasound antenatally and may show significant spontaneous improvement postnatally. However, this cannot be easily predicted, and the postnatal course and severity of 'benign prenatal' HPP ranges from that observed in patients with infantile HPP to odonto-HPP. ${ }^{4}$

While early tooth loss (due to failure of normal cementum production) is a wellrecognised presentation in less severely affected older children, the effect of HPP on motor function is less recognised but in some cases debilitating. Similarly, adolescents and young adults can suffer severe loss of their secondary dentition and some may develop stress fractures, particularly of metatarsals, that heal slowly and lead to progressive loss of mobility.

Because HPP presentation is so variable, patients can present initially to different healthcare specialists (eg, obstetrician/neonatologist, paediatrician, dentist, general practitioner, orthopaedic surgeon), making recognition of the clinical symptoms essential for rapid diagnosis and access to treatment. We have developed a diagnostic pathway to enable recognition of symptoms and minimise misdiagnosis (figure 1).

Clinical findings of the most common manifestations are shown in figure 1; note that presentation can be multisystemic. Low ALP activity level is the hallmark of HPP and should be assessed when HPP is suspected. Measurement of ALP is easy to perform, readily available and can lead to rapid diagnosis. However, its interpretation requires some care. The normal range for ALP is age- and gender-specific, emphasising the need for appropriate laboratory reference ranges. ${ }^{5}$ Some laboratories only have one broad reference range for all ages; physicians should be wary because ALP levels that are normal for adults could represent pathological levels for children. Furthermore, there is often a focus upon elevated ALP for the diagnosis of rickets/osteomalacia, rather than low ALP levels. ${ }^{6}$

Together with low ALP levels, typical radiological features of perinatal, infantile and childhood HPP can confirm the diagnosis. However, the radiological features in mild childhood, adult and odonto-HPP are not always diagnostic. Where the diagnosis is in doubt, confirmatory laboratory testing can include PLP, PPi or urinary phosphoethanolamine levels, which are usually above the reference range in patients with $\mathrm{HPP}^{5}{ }^{6}$ as well as $A L P L$ mutational analyses.

The presence of any clinical findings shown in figure 1 with a combination of low ALP activity level should result in a working diagnosis of HPP until proven otherwise. Timely access to care and treatment options is dependent on the critical period between symptom onset and diagnosis. This is especially important for patients with perinatal and infantile HPP, where delays may have fatal consequences, or in older patients where 


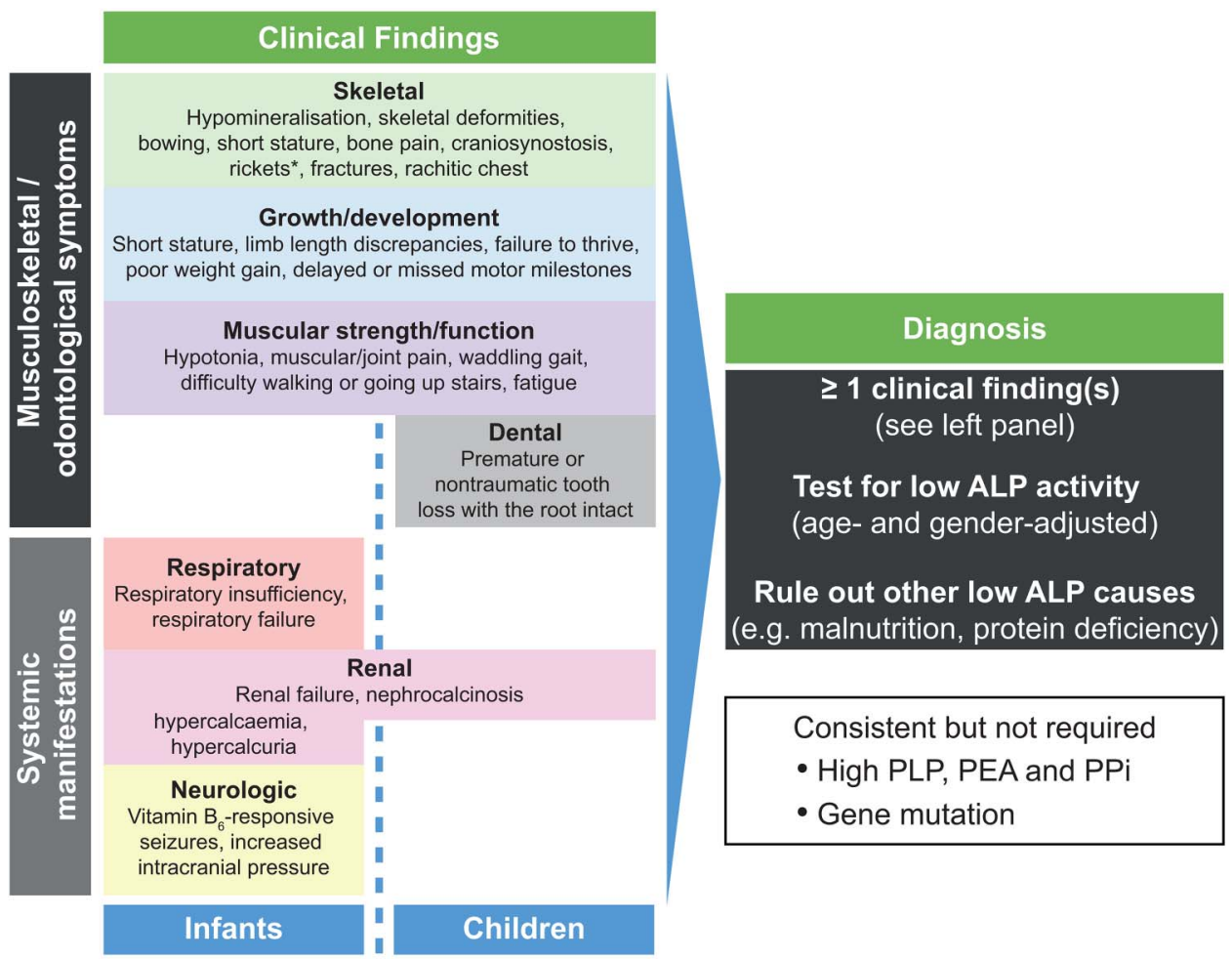

Figure 1 Hypophosphatasia (HPP) diagnostic pathway. This pathway focuses on presentation and diagnosis of infants and children with HPP. *Radiological findings can include profound hypomineralisation, irregularity of the provisional zone of calcification, physeal widening, metaphyseal flaring/fraying, metaphyseal radiolucencies and fractures. ${ }^{13}$ ALP, alkaline phosphatase; PEA, phosphoethanolamine; PLP, pyridoxal 5'-phosphate; PPi, inorganic pyrophosphate.

treatment with bisphosphonates may worsen the bone phenotype. Improving awareness will increase the likelihood of a correct diagnosis and minimise the period between symptom onset and initiating the newly available, potentially life-saving therapy.

We hope that despite the inconvenience of frequent injections and the high cost of the treatment, this transformative therapy will be implemented for those in whom HPP is currently life-limiting.

Funding Editorial support was provided by Fishawack Communications $\mathrm{GmbH}$ and was funded by Alexion Pharmaceuticals Inc

Competing interests NB has received fees for contractual work and honoraria from Alexion Pharmaceuticals Inc. CFM has received consultancy fees, grants and honoraria from Alexion Australia. KO has received honoraria from Alexion Pharmaceuticals Inc.

Provenance and peer review Commissioned; externally peer reviewed.

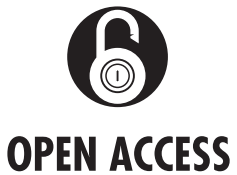

Open Access This is an Open Access article distributed in accordance with the Creative Commons Attribution Non Commercial (CC BY-NC 4.0) license, which permits others to distribute, remix, adapt, build upon this work non-commercially, and license their derivative works on different terms, provided the original work is properly cited and the use is non-commercial. See: http:// creativecommons.org/licenses/by-nc/4.0/

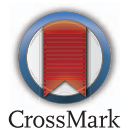

To cite Bishop N, Munns CF, Ozono K. Arch Dis Child 2016;101:514-515.

Received 16 December 2015

Revised 18 February 2016
Accepted 25 February 2016

Published Online First 18 March 2016

Arch Dis Child 2016:101:514-515.

doi:10.1136/archdischild-2015-309579

\section{REFERENCES}

1 Whyte MP. Hypophosphatasia. In: Genetics of bone biology and skeletal disease. In: Thakker RV, Whyte MP, Eisman J, Igarashi T. eds. Academic press, 2013:337-60

2 Okazaki Y, Kitajima $\mathrm{H}$, Mochizuki N, et al. Lethal hypophosphatasia successfully treated with enzyme replacement from day 1 after birth. Eur I Pediatr 2016;175:433-7.

3 Whyte MP, Rockman-Greenberg C, Ozono K, et al. Asfotase alfa treatment improves survival for perinatal and infantile hypophosphatasia. I Clin Endocrinol Metab 2016:101:334-42.

4 Wenkert D, McAlister WH, Coburn SP, et al. Hypophosphatasia: nonlethal disease despite skeletal presentation in utero (17 new cases and literature review). J Bone Miner Res 2011;26:2389-98.

5 Mornet E, Nunes ME. Hypophosphatasia. In: Pagon RA, Adam MP, Ardinger HH, et al, eds. University of Washington, GeneReviews(R). Seattle, WA, 1993.

6 Rockman-Greenberg C. Hypophosphatasia. Pediatr Endocrinol Rev 2013;10(Suppl 2):380-8. 